\title{
Spectroscopic evidence of multiple populations in globular clusters
}

\author{
R. Gratton ${ }^{1}$, S. Lucatello ${ }^{1}$, E. Carretta ${ }^{2}$ and A. Bragaglia ${ }^{2}$ \\ ${ }^{1}$ INAF-Osservatorio Astronomico di Padova \\ ${ }^{2}$ INAF-Osservatorio Astronomico di Bologna
}

\begin{abstract}
We review spectroscopic evidence of multiple stellar populations in globular clusters. First, we lay down the basic data: the $\mathrm{C}-\mathrm{N}, \mathrm{Na}-\mathrm{O}, \mathrm{Mg}-\mathrm{Al}$ anti-correlations among red giants and main sequence stars, and discuss how they appear to be general properties of globular clusters, in spite of cluster-to-cluster differences. We will then describe what is currently known about He from spectroscopy. We will then present the implications and current observations for the interpretation of the horizontal branches, showing that the multiple population phenomenon is strongly related to the distribution of stars along them. We will briefly mention the spectroscopic evidence related to some less understood cases, like the clusters with multiple subgiant branches. Finally, we summarize the relation between multiple populations and general properties for globular clusters, and their implications for the formation scenario.
\end{abstract}

Keywords. (Galaxy:) globular clusters: general, stars: abundances, Galaxy: halo

The last ten years have seen a revolution in our concept of globular clusters (GCs) thanks to data coming from new efficient multi-object spectrographs on 8-m telescopes (e.g. Carretta et al. 2009a, b) and the exquisite photometric precision of HST (e.g. Bedin et al. 2004). These studies clarified the meaning of large star-to-star abundance variations for several light elements known to be present in GCs for almost forty years (see Gratton et al. 2004 and 2012 for recent reviews) and revealed multiple sequences on the red giant branch (RGB), subgiant branch (SGB), and main sequence (MS) in several GCs. Regarded for a long time as intriguing abundance anomalies restricted to some cluster stars, the peculiar chemical patterns only recently were explicitly understood as a universal phenomenon in GCs related to their very same nature/origin (Carretta 2006; Carretta et al. 2006, 2009a, b, 2010). The observational pattern is quite well assessed (see the reviews by Gratton et al. 2004, 2012, and Piotto 2009), thanks to several important milestones:

(i) Abundance variations of the heavier species ( $\mathrm{O}-\mathrm{Na}$ and $\mathrm{Mg}-\mathrm{Al}$ anti-correlations) are restricted to the denser cluster environment. Evolutionary changes along the RGB additionally affect other light elements (Li, C, and N: Charbonnel et al. 1998; Gratton et al. 2000).

(ii) This pattern is established in $p$-capture reactions of the $\mathrm{CNO}, \mathrm{NeNa}$ and $\mathrm{MgAl}$ chains in high temperature H-burning (Denisenkov \& Denisenkova 1989).

(iii) The variations are found also among unevolved stars currently on the MS of GCs (Briley et al. 1996; Gratton et al.2001). This calls for a class of now-extinct stars, more massive than the low-mass ones presently evolving in GCs, as the site for the nucleosynthesis.

(iv) The observed variations in the abundances of $p$-capture elements are connected to that of He, i.e., stars that are Na-rich and O-poor are also He-rich, although the relation between these abundance variations may be complicated. Multiple MSs, attributed to populations with different He fraction $\mathrm{Y}$, have been found in some GCs (Bedin et al. 
2004, Piotto et al. 2007). There are indications that $\mathrm{Y}$ differences can be traced also on the RGB (Carretta et al. 2007, Bragaglia et al. 2010, Pasquini et al. 2011), and contribute to explaining the horizontal branch (HB) second parameter problem (Gratton et al. 2010).

In summary, in contrast with the common assumption, GCs are not simple stellar populations: they harbour various stellar generations, distinct by their chemistry. These populations may be separated using the patterns of anti-correlated $\mathrm{Na}-\mathrm{O}, \mathrm{Mg}-\mathrm{Al}$ or photometric methods. They provide insights into the early phases of GCs, since the time scale for the release of matter processed by H-burning at high temperatures is less than $10^{8}$ year. To decipher the relevant information, large and homogeneous datasets are needed, like the one recently gathered by Carretta et al. (2009a, 2009b), from which Carretta et al. (2010) were able to place the properties of different stellar generations in GCs and global cluster parameters into a general framework for the formation of GCs. The Na-O anti-correlation may be used to separate GCs (hosting multiple stellar populations) from open clusters (OCs, where a single stellar population is present), and first and second generation (FG and SG) stars within each GC. The emerging scenario for the formation of GCs includes various phases: a large episode of star formation (the FG) and then the formation of the current GC (mainly made of SG stars) within a cooling flow formed by the slow winds of a fraction of this FG (D'Ercole et al. 2008). Some stars of the FG remain in the cluster. Since the ejecta of only a small fraction of the FG stars are used to form the SG, a large fraction of the FG must have been lost by the proto-GCs and may represent the main component of halo field stars (see also Martell \& Grebel 2010; Vesperini et al. 2010; Schaerer \& Charbonnel 2011). This links directly the formation of GC to that of galaxies.

\section{References}

Bedin, L. R., et al., 2004, ApJ, 605, L125

Bragaglia, A., et al., 2010, A\& $A, 519,60$

Briley, M., et al., 1996, Nature, 383, 604

Charbonnel, C., et al., 1998, A\&GA, 332, 204

Carretta E., 2006, AJ, 131, 1766

Carretta, E., et al., 2006, A\&SA, 450, 523

Carretta, E., et al., 2007, A\&SA, 464, 927

Carretta, E., et al., 2009a, A\&A A, 505, 117

Carretta, E., et al., 2009b, A\&AA, 505, 139

Carretta, E., et al.2010, A\&A $A, 516,55$

Denisenkov, P. A. \& Denisenkova, S. N. 1989, A.Tsir., 1538, 11

D'Ercole, A., et al. 2008, MNRAS, 391, 825

Gratton, R. G., et al., 2000, $A \mathscr{\mho} A, 358,671$

Gratton, R. G., et al., 2001, $A \& A$, 369, 87

Gratton, R. G., et al., 2004, ARA $\& A, 42,38$

Gratton, R. G., et al., 2010, $A \mathscr{E} A, 517,8$

Gratton, R. G., et al., 2012, $A \mathscr{E} A R v, 20,50$

Martell, S. L. \& Grebel, E. K. 2010, A\& $A$, 519, 14

Pasquini, L., et al., 2011, A\&A, 531, 35

Piotto, G. 2009, in IAUS 258, 233

Piotto, G., et al., 2007, ApJ, 661, L53

Schaerer, D. \& Charbonnel, C., 2011, MNRAS 413, 2297

Vesperini, E., et al., 2010, ApJ, 718, L112 